\title{
Geometric and Topological Methods in Computer Science (special 10th anniversary GETCO conference, 2018)
}

\author{
Sergio Rajsbaum ${ }^{1} \cdot$ Shmuel Weinberger ${ }^{2}$ \\ Published online: 13 February 2020 \\ (C) Springer Nature Switzerland AG 2020
}

We are happy to present this special issue of the Journal of Applied and Computational Topology devoted to applications within computer science. The Geometric and Topological Methods in Computer Science (GETCO) conference series focus on applications of algebraic topology in computer science with special emphasis in concurrency, distributed computing, networking and other situations related to systems of sequential computers that communicate with each other. This special issue of the Journal of Applied and Computational Topology celebrates the 10th GETCO conference.

The first GETCO conference was held in Aalborg in 1999. Applications of algebraic topology in concurrency was then a new subject, fostered by seminal papers such as those by Vaughn Pratt in ACM POPL 1991 and Eric Goubault in CONCUR 1992 and CONCUR 1993, on the formal methods side, and the ACM STOC 1993 papers by Herlihy-Shavit and Saks-Zaharoglou on the distributed computing side, and were publicized by the Workshop on New Connections between Mathematics and Computer Science, organized by Jeremy Gunawardena in Cambridge Nov. 1995. The seven succeeding GETCO workshops were held as satellites to CONCUR or DISC. These were held at Penn State University in 2000, Aalborg in 2001, Toulouse in 2002, Marseille in 2003, Amsterdam 2004, San Francisco in 2005, Bonn in 2006.

The 2010 workshop had a broader scope and included further applications of algebraic topology including robotics and shape analysis. GETCO was back in Aalborg for its 9th edition in 2015, and also included some topological data analysis. Since then, a new European (ESF) network ACAT, Applied and Computational Algebraic Topology, has been established, and two books have been published Distributed Computing Through Combinatorial Topology and Directed Algebraic Topology and Concurrency; some of many indications that

Sergio Rajsbaum

rajsbaum@im.unam.mx

1 UNAM, Mexico City, Mexico

2 University of Chicago, Chicago, USA 
applications of algebraic topology to concurrent systems is now a mature subject, widespread and with impact in many fields. And now in its 10 th edition with GETCO 2018, expanding further to neuroscience and learning applications.

The 10th GETCO took place in Oaxaca, Mexico, September 10-14, 2018. The Scientific Committee included:

Armando Castañeda, UNAM, Mexico,

Dmitry Feichtner-Kozlov, University of Bremen, Germany,

Eric Goubault, Ecole Polytechnique, France,

Maurice Herlihy, Brown University, USA,

Ran Levi, University of Aberdeen, UK,

Sergio Rajsbaum, UNAM, Mexico, and

Martin Raussen, Aalborg University, Denmark.

The conference included a full day of tutorials:

Directed algebraic topology by Martin Raussen, Aalborg University.

Combinatorial topology and distributed computing by Sergio Rajsbaum, UNAM.

Combinatorial topology by Dmitry Feichtner-Kozlov, University of Bremen.

Topology in neural networks by Ran Levi, University of Aberdeen.

and twenty five scientific talks over 4 days, with speakers from Australia, Canada, Denmark, France, Germany, Israel, Italy, Mexico, Poland, Portugal, Slovenia, Switzerland, UK, and USA. The full program can be found at https://sites.google. com/view/geometricandtopologicalmethods/home.

Participants were invited to submit contributions to this special issue. The eight papers that were accepted went through the usual, rigorous reviewing process of the Journal of Applied and Computational Topology, a journal that aims to foster the application of geometry and topology to other fields, and to the development of the computational tools that foster this. We are happy to offer herewith this special issue.

Publisher's Note Springer Nature remains neutral with regard to jurisdictional claims in published maps and institutional affiliations. 